\title{
openheart Renal denervation and blood pressure reduction in resistant hypertension: a systematic review and meta-analysis
}

\author{
Chun Shing Kwok, ${ }^{1}$ Yoon K Loke, ${ }^{2}$ Shiva Pradhan, ${ }^{3}$ Bernard Keavney, ${ }^{1}$ \\ Magdi El-Omar, ${ }^{4}$ Mamas A Mamas ${ }^{1,3}$
}

To cite: Kwok CS, Loke YK, Pradhan S, et al. Renal denervation and blood pressure reduction in resistant hypertension: a systematic review and meta-analysis. Open Heart 2014;1:e000092. doi:10.1136/openhrt-2014000092

- Additional material is available. To view please visit the journal (http://dx.doi.org/ 10.1136/openhrt-2014000092).

Received 26 February 2014 Revised 11 May 2014 Accepted 15 July 2014

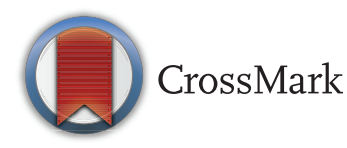

${ }^{1}$ Cardiovascular Institute, University of Manchester, Manchester, UK

${ }^{2}$ Norwich Medical School, University of East Anglia, Norwich, Norfolk, UK ${ }^{3}$ Leeds General Infirmary, Great George Street, Leeds, UK

${ }^{4}$ Manchester Heart Centre, Manchester Royal Infirmary, Manchester, UK

Correspondence to Dr Chun Shing Kwok; shingkwok@doctors.org.uk

\section{ABSTRACT}

Objective: The objective of this study is to evaluate the efficacy and safety of renal denervation in patients with resistant hypertension.

Methods: We searched MEDLINE and EMBASE for studies that evaluated the use of catheter-based renal sympathetic denervation compared to a control group and reported blood pressure results at follow-up. Data was extracted from relevant studies and pooled estimates for blood pressure were determined using the inverse variance method for meta-analysis with mean difference.

Results: We identified 12 studies (three randomised controlled trials $(n=688)$, eight prospective observational studies $(n=478)$ and one observational study with matched controls $(n=310)$ ). Data from SYMPLICITY HTN-3, the only high-quality blinded randomised control trial suggests that there is no significant difference in change in systolic $(-2.3095 \% \mathrm{Cl}-6.90$ to $2.30 \mathrm{~mm} \mathrm{Hg})$ or diastolic $(-1.9695 \% \mathrm{Cl}-4.98$ to $1.06 \mathrm{~mm} \mathrm{Hg})$ blood pressure at 6 months. The pooled data from two unblinded trials of lower quality showed significant reduction in change in systolic $(-27.3695 \% \mathrm{Cl}-37.08$ to $-24.61 \mathrm{~mm} \mathrm{Hg}$ ) and diastolic blood pressure $(-9.62$ $95 \% \mathrm{Cl}-14.51$ to $-4.72 \mathrm{~mm} \mathrm{Hg}$ ). In terms of safety, SYMPLICITY HTN-3 found no significant differences between treatment and control group in terms of death, myocardial infarction, new onset renal disease, stroke and hypertensive emergencies.

Conclusions: In conclusion, while poor quality unblinded studies provide evidence that renal denervation using catheter-based systems is effective in reducing systolic and diastolic blood pressure in resistant hypertension, the largest randomised controlled trial to date (SYMPLICITY HTN-3) failed to demonstrate any benefit.

\section{INTRODUCTION}

Hypertension is an important risk factor for mortality worldwide, causing an estimated 7.5 million deaths per year. ${ }^{1}$ Despite receiving hypertensive medications, only 53\% of patients with hypertension achieve the recommended blood pressure targets, ${ }^{2}$ with

\section{KEY MESSAGES}

What is already known about the subject?

- Renal denervation has generate significant interest as a method for the treatment of systemic hypertension.

- Several non-blinded studies of renal denervation have shown favourable reductions in blood pressure.

What does this study add?

- The high quality SIMPLICITY HTN-3 trial, failed to demonstrate any significant improvement in blood pressure compared to control, whereas meta-analysis of two RCTs without sham control or blinding found significant reduction in $\mathrm{BP}$ with renal denervation.

- Other non-blinded observational studies which are at risk of bias appear to suggest that there are significant reduction in blood pressure with renal denervation.

How might this impact on clinical practice?

- Current evidence provides insufficient evidence to support the use of renal denervation in the treatment of resistant hypertension as the highest quality trial failed to demonstrates this relationship.

a proportion of these patients developing resistant hypertension. Resistant hypertension is defined as blood pressure that remains above goal in spite of the concurrent use of three antihypertensive agents of different classes, with one of the three agents being a diuretic. ${ }^{3}$

There has been significant interest in targeting the renal sympathetic nervous system in treatment of systemic hypertension. Evidence suggests that sympathetic nervous system over-activity is responsible for the development and maintenance of hypertension. $^{4}$ Historical observations have shown that surgical sympathectomy can achieve good blood pressure reduction. ${ }^{5}$ More 
recently, catheter-based renal denervation that applies low-level radiofrequency (RF) energy to disrupt renal sympathetic nerves within the renal artery wall has emerged as a promising minimally invasive treatment for hypertension. There is growing clinical evidence to suggest that this method effectively and safely reduces blood pressure in patients with resistant hypertension. ${ }^{6} 7$ Most recently, however, the SYMPLICITY HTN-3 single blind randomised controlled study reported a failure to reach its primary efficacy endpoint of a reduction in office-based systolic blood pressure from baseline to 6 months. ${ }^{89}$

In view of the renewed interest around renal denervation, particularly in light of the SYMPLICITY HTN-3 trial data, we report a systematic review and meta-analysis, which aims to evaluate the efficacy of renal denervation in the treatment of resistant hypertension over time.

\section{METHODS}

\section{Study eligibility}

Studies were considered for inclusion if they evaluated the use of catheter-based renal sympathetic denervation compared to a control group and reported blood pressure results at follow-up. Single arm studies, case reports, case series, letters and editorials were excluded, but relevant reviews were retrieved to identify additional studies.

\section{Search strategy}

Our search was carried out using the OvidSP interface covering MEDLINE and EMBASE from inception until April 2014. Brown search terms were used to reduce likelihood of missing relevant studies (see online supplementary appendix 1$)$.

Two reviewers (CSK and SP) independently checked retrieved titles and abstracts for eligibility, and the relevant abstracts were checked by the other reviewers (YKL and MAM). Finally, two reviewers manually searched bibliographies of included studies, as well as full-text review articles identified from the search (CSK and YKL).

\section{Data extraction}

Two reviewers (CSK and SP) extracted data on study, design, patient characteristics, treatment, follow-up and results, and performed quality assessment of included studies. This was checked by the other reviewers (MAM and YKL).

\section{Data synthesis}

We planned to perform meta-analysis using RevMan V.5.1.2 (Nordic Cochrane Centre) using the inverse variance method for mean difference if there was not more than a moderate degree of heterogeneity. The random effects model was used because it considers study heterogeneity when generating an average estimate. Statistical heterogeneity was evaluated through the $\mathrm{I}^{2}$ statistic where values of $30-60 \%$ were representing moderate heterogeneity. ${ }^{10}$

\section{Validity assessment}

Validity assessment was performed by considering use of blinding, outcome ascertainment, baseline differences, loss to follow-up and selective reporting. A subjective overall risk of bias was also assigned for each study based on these factors. In addition, we planned to conduct asymmetry testing for publication bias provided that there were $>10$ studies in the meta-analysis and if statistical heterogeneity was $<50 \% .^{11}$

\section{RESULTS}

Twelve studies met the inclusion criteria (study selection is shown in online supplementary appendix 2). These studies included three randomised controlled trials, ${ }^{7-9} 12$ eight prospective observational studies ${ }^{6}{ }^{13-19}$ and one observational study with matched controls ${ }^{20}$ with a total of 1556 participants (table 1). In general, the participant selection criteria were similar across all but one study that randomised patients with atrial fibrillation to renal denervation and pulmonary isolation versus pulmonary isolation alone. ${ }^{12}$

The risk of bias assessment is shown in online supplementary appendix 3. All included studies were nonblinded except for SYMPLICITY HTN-3. The majority of studies were deemed to be of at least moderate risk of bias. Two studies were only available in abstract form and were deemed to be at high risk of bias.

The renal denervation procedures, control group, follow-up and results are shown in table 2. The majority of studies used the SYMPLICITY catheter system by Medtronic with multiple ablations in both renal arteries. All except two studies did not describe in detail the management received by the control group. In the matched observational study, ${ }^{20}$ normotensive and controlled blood pressure controls from the Australian Diabetes, Obesity and Lifestyle database were used. In another randomised trial of patients with atrial fibrillation, patients were randomised to pulmonary vein isolation alone or in combination with renal denervation. All studies included reported increased reductions in systolic and diastolic blood pressure with renal denervation therapy, compared to controls.

Three randomised controlled trials were considered for meta-analysis. However, there was significant statistical heterogeneity when pooling the three randomised controlled trials and it was decided that the two unblinded trials (of moderate-high risk of bias) would be considered separately from the higher quality trial that had used a sham procedure as placebo. Data from SYMPLICITY HTN-3, the only high-quality blinded randomised control trial, suggests that there was no significant difference in change in systolic $(-2.3095 \%$ CI -6.90 to $2.30 \mathrm{~mm} \mathrm{Hg})$ or diastolic $(-1.9695 \% \mathrm{CI}-4.98$ to $1.06 \mathrm{~mm} \mathrm{Hg}$ ) blood pressure at 6 months. The pooled 
Table 1 Study design, patient characteristics of parallel group studies of renal denervation versus control in resistant hypertension

\begin{tabular}{|c|c|c|c|c|c|c|c|}
\hline & Design & Year & Country & $\begin{array}{l}\text { Number } \\
\text { of participants } \\
\text { (treatment, control) }\end{array}$ & Mean age & $\begin{array}{l}\text { Per cent } \\
\text { male }\end{array}$ & Selection criteria and management \\
\hline \multicolumn{8}{|l|}{ Clinical trials } \\
\hline $\begin{array}{l}\text { Pokushalov } \\
\text { et } a l^{12}\end{array}$ & Randomised trial & NA & $\begin{array}{l}\text { Russia, The } \\
\text { Netherlands and } \\
\text { USA }\end{array}$ & $27(13,14)$ & $\begin{array}{l}56 \text { and } \\
57 \text { years }\end{array}$ & 78 & $\begin{array}{l}\text { Drug-refractory AF or paroxysmal AF, with } \\
\text { BP } \geq 160 \mathrm{~mm} \mathrm{Hg} \mathrm{(} \geq 150 \mathrm{~mm} \mathrm{Hg} \text { with type } 2 \\
\text { diabetes) with at least } 3 \text { antihypertensive } \\
\text { drugs and eGFR } \geq 45 \mathrm{~mL} / \mathrm{min} / 1.73 \mathrm{~m}^{2} \text {. } \\
\text { Diuretics were used in } 96 \% \text { of patients. } \\
\text { Patients with secondary causes of } \\
\text { hypertension were excluded }\end{array}$ \\
\hline $\begin{array}{l}\text { SYMPLICITY } \\
\text { HTN-2 }^{7}\end{array}$ & Randomised trial & $\begin{array}{l}\text { June } 2009 \text { to } \\
\text { January } 2010\end{array}$ & $\begin{array}{l}\text { Europe, } \\
\text { Australia and } \\
\text { New Zealand }\end{array}$ & $106(52,54)$ & 58 years & 58 & $\begin{array}{l}\text { Age }>18 \text { years with } \mathrm{BP} \geq 160 \mathrm{~mm} \mathrm{Hg} \\
(\geq 150 \mathrm{~mm} \mathrm{Hg} \text { with type } 2 \text { diabetes) with at } \\
\text { least } 3 \text { antihypertensive drugs. Diuretics } \\
\text { were used in } 90 \% \text { of patients. Patients with } \\
\text { significant renal artery stenosis or renal } \\
\text { artery anatomy that precluded treatment } \\
\text { were excluded }\end{array}$ \\
\hline $\begin{array}{l}\text { SYMPLICITY } \\
\text { HTN-3 }{ }^{8}\end{array}$ & Randomised trial & $\begin{array}{l}\text { September } \\
2011 \text { to } \\
\text { January } 2014\end{array}$ & International & 535 & 57 years & 61 & $\begin{array}{l}\text { Age } \geq 18 \text { and } \leq 80 \text { years on stable medical } \\
\text { regimen of } \geq 3 \text { antihypertensives. Office BP } \\
\geq 160 \mathrm{~mm} \mathrm{Hg} \text { on average of } 3 \text { readings on } \\
\text { initial screening and confirmatory screening. } \\
\text { Exclusions: eGFR }<45 \mathrm{~mL} / \mathrm{min} / 1.73 \mathrm{~m}^{2}, \\
\text { ambulatory blood pressure monitoring } \\
\text { average }<135 \mathrm{~mm} \mathrm{Hg} \text {, type } 1 \text { diabetes, } \\
\text { chronic oxygen support, mechanical } \\
\text { ventilation, primary pulmonary hypertension } \\
\text { or pregnancy }\end{array}$ \\
\hline \multicolumn{8}{|c|}{ Observational studies } \\
\hline Brandt et al ${ }^{14}$ & $\begin{array}{l}\text { Prospective } \\
\text { observational } \\
\text { study }\end{array}$ & $\begin{array}{l}\text { October } 2009 \\
\text { to January } \\
2011\end{array}$ & $\begin{array}{l}\text { Austria and } \\
\text { Germany }\end{array}$ & $64(46,18)$ & $\begin{array}{l}63 \text { years in } \\
\text { both } \\
\text { groups }\end{array}$ & 67 and 61 & $\begin{array}{l}\text { Patients age }>18 \text { years with } \mathrm{BP} \geq 160 \mathrm{~mm} \mathrm{Hg} \\
\text { ( } \geq 150 \mathrm{~mm} \mathrm{Hg} \text { with type } 2 \text { diabetes) with at } \\
\text { least } 3 \text { antihypertensive drugs. All patients } \\
\text { were on diuretic treatment. Patients with } \\
\text { secondary causes of hypertension were } \\
\text { excluded }\end{array}$ \\
\hline Brandt et $a l^{15}$ & $\begin{array}{l}\text { Prospective } \\
\text { observational } \\
\text { study }\end{array}$ & $\begin{array}{l}\text { October } 2009 \\
\text { to September } \\
2011\end{array}$ & $\begin{array}{l}\text { Austria and } \\
\text { Germany }\end{array}$ & $120(110,10)$ & $\begin{array}{l}64 \text { and } \\
65 \text { years }\end{array}$ & 70 and 80 & $\begin{array}{l}\text { Patients age }>18 \text { years with } \mathrm{BP} \geq 160 \mathrm{~mm} \mathrm{Hg} \\
\text { ( } \geq 150 \mathrm{~mm} \mathrm{Hg} \text { with type } 2 \text { diabetes) with at } \\
\text { least } 3 \text { antihypertensive drugs. Diuretics } \\
\text { were used in } 83 \% \text { of patients. Patients with } \\
\text { secondary causes of hypertension were } \\
\text { excluded }\end{array}$ \\
\hline Fatum et al ${ }^{16}$ & & NA & Germany & $21(15,6)$ & $\begin{array}{l}67 \text { and } \\
61 \text { years }\end{array}$ & 59 and 60 & $\begin{array}{l}\text { Patients age }>18 \text { years with } \mathrm{BP} \geq 160 \mathrm{~mm} \mathrm{Hg} \\
\text { ( } \geq 150 \mathrm{~mm} \mathrm{Hg} \text { with type } 2 \text { diabetes) with at }\end{array}$ \\
\hline
\end{tabular}


Table 1 Continued

\begin{tabular}{|c|c|c|c|c|c|c|c|}
\hline & Design & Year & Country & $\begin{array}{l}\text { Number } \\
\text { of participants } \\
\text { (treatment, control) }\end{array}$ & Mean age & $\begin{array}{l}\text { Per cent } \\
\text { male }\end{array}$ & Selection criteria and management \\
\hline & $\begin{array}{l}\text { Prospective } \\
\text { observational } \\
\text { study }\end{array}$ & & & & & & $\begin{array}{l}\text { least } 3 \text { antihypertensive drugs. Unclear use } \\
\text { of diuretics. Unclear if patients with } \\
\text { secondary causes of hypertensions were } \\
\text { included }\end{array}$ \\
\hline Franzen et al ${ }^{13}$ & $\begin{array}{l}\text { Prospective } \\
\text { observational } \\
\text { study }\end{array}$ & NA & Germany & $27(21,6)$ & 63 years & NA & $\begin{array}{l}\text { Patients with } \mathrm{BP} \geq 150 \mathrm{~mm} \mathrm{Hg} \text { with at least } 3 \\
\text { antihypertensive drugs. All patients were on } \\
\text { diuretic treatment. Patients with secondary } \\
\text { causes of hypertensions were excluded }\end{array}$ \\
\hline Krum et $a^{\rho}$ & $\begin{array}{l}\text { Prospective } \\
\text { observational } \\
\text { study }\end{array}$ & $\begin{array}{l}\text { June } 2007 \text { to } \\
\text { November } \\
2008\end{array}$ & $\begin{array}{l}\text { Australia and } \\
\text { Europe }\end{array}$ & $50(45,5)$ & 57 years & 58 & $\begin{array}{l}\text { Patients age }>18 \text { years with } \mathrm{BP} \geq 160 \mathrm{~mm} \mathrm{Hg} \\
\text { ( } \geq 150 \mathrm{~mm} \mathrm{Hg} \text { with type } 2 \text { diabetes) with at } \\
\text { least } 3 \text { antihypertensive drugs. Diuretics } \\
\text { were used in } 96 \% \text { of patients. Patients with } \\
\text { secondary causes of hypertensions were } \\
\text { excluded }\end{array}$ \\
\hline Lambert et a ${ }^{20}$ & $\begin{array}{l}\text { Observational } \\
\text { study with match } \\
\text { controls }\end{array}$ & NA & Australia & $\begin{array}{l}62 \text { treatment, } 248 \\
\text { controls }\end{array}$ & 62 years & 65 & $\begin{array}{l}\text { Patients with resistant hypertension. } \\
\text { Diuretics were used in } 85 \% \text { of patients. } \\
\text { Unclear if patients with secondary causes of } \\
\text { hypertension were excluded }\end{array}$ \\
\hline Mahfoud et al ${ }^{17}$ & $\begin{array}{l}\text { Prospective } \\
\text { observational } \\
\text { study }\end{array}$ & $\begin{array}{l}\text { March } 2009 \text { to } \\
\text { May } 2010\end{array}$ & $\begin{array}{l}\text { Australia and } \\
\text { Germany }\end{array}$ & $50(37,13)$ & 60 years & 74 & $\begin{array}{l}\text { Patients with age }>18 \text { years with BP } \\
\geq 160 \mathrm{~mm} \mathrm{Hg} \mathrm{(} \geq 150 \mathrm{~mm} \mathrm{Hg} \text { with type } 2 \\
\text { diabetes) with at least } 3 \text { antihypertensive } \\
\text { drugs. All patients were taking diuretics. } \\
\text { Patients with renal artery abnormality or } \\
\text { stenosis were excluded }\end{array}$ \\
\hline Mahfoud et $a l^{18}$ & $\begin{array}{l}\text { Prospective } \\
\text { observational } \\
\text { study }\end{array}$ & $\begin{array}{l}\text { January } 2010 \\
\text { to February } \\
2011\end{array}$ & $\begin{array}{l}\text { Australia and } \\
\text { Germany }\end{array}$ & $100(88,12)$ & 62 years & 61 & $\begin{array}{l}\text { Patients with age }>18 \text { years with BP } \\
\geq 160 \mathrm{~mm} \mathrm{Hg} \mathrm{(} \geq 150 \mathrm{~mm} \mathrm{Hg} \text { with type } 2 \\
\text { diabetes) with at least } 3 \text { antihypertensive } \\
\text { drugs. All patients had received diuretics. } \\
\text { Patients with renal artery abnormality or } \\
\text { stenosis were excluded }\end{array}$ \\
\hline Ukena et al ${ }^{19}$ & $\begin{array}{l}\text { Prospective } \\
\text { observational } \\
\text { study }\end{array}$ & $\begin{array}{l}\text { March } 2009 \text { to } \\
\text { October } 2010\end{array}$ & $\begin{array}{l}\text { Germany, } \\
\text { Australia, USA }\end{array}$ & $\begin{array}{l}46(37,9) .28 \text { from } \\
\text { SYMPLICITY HTN-2 }\end{array}$ & 60 years & 70 & $\begin{array}{l}\text { Patients with age }>18 \text { years with BP } \\
\geq 160 \mathrm{~mm} \mathrm{Hg} \text { ( } \geq 150 \mathrm{~mm} \mathrm{Hg} \text { with type } 2 \\
\text { diabetes) with at least } 3 \text { antihypertensive } \\
\text { drugs. Diuretics were used in } 87 \% \text { of } \\
\text { patients. Patients with secondary causes of } \\
\text { hypertension were excluded }\end{array}$ \\
\hline
\end{tabular}


Table 2 Treatment, follow-up and results of parallel group studies of renal denervation versus control in resistant hypertension

\begin{tabular}{|c|c|c|c|c|}
\hline Clinical trials & RD group & Control group & $\begin{array}{l}\text { Duration of } \\
\text { follow-up }\end{array}$ & Efficacy results \\
\hline $\begin{array}{l}\text { Pokushalov } \\
\text { et } a l^{12}\end{array}$ & $\begin{array}{l}\text { RFA of } 8-10 \mathrm{~W} \text { for } 2 \text { min up to } 6 \text { lesions } \\
\text { and pulmonary vein isolation }\end{array}$ & Pulmonary vein isolation alone & 12 months & $\begin{array}{l}\text { Reduction at } 6 \text { months } \\
\text { Systolic BP } \\
\text { Denervation: }-28 \pm 7 \mathrm{~mm} \mathrm{Hg} \\
\text { Control: }-5 \pm 5 \mathrm{~mm} \mathrm{Hg} \\
\text { Diastolic BP: } \\
\text { Denervation: }-10 \pm 6 \mathrm{~mm} \mathrm{Hg} \\
\text { Control: }-3 \pm 6 \mathrm{~mm} \mathrm{Hg}\end{array}$ \\
\hline 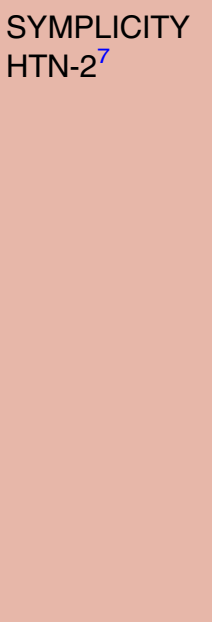 & $\begin{array}{l}\text { Catheter-based RD with SYMPLICITY } \\
\text { Catheter System }\end{array}$ & Continuation of anti-hypertensive drugs & $\begin{array}{l}1,3 \text { and } \\
6 \text { months }\end{array}$ & $\begin{array}{l}\text { Systolic BP } \\
\text { RD group }(n=52) \text { : } \\
\text { Baseline } 178 \pm 18 / 96 \pm 16 \mathrm{~mm} \mathrm{Hg} \text {. Change at } \\
1 \text { months }-20,3 \text { months }-24,6 \text { months }-32 \\
\pm 23 \mathrm{~mm} \mathrm{Hg} \text {. } \\
\text { Control group ( } n=54 \text { ): Baseline mm Hg. Change } \\
\text { at } 1 \text { months } 0,3 \text { months }-4,6 \text { months } 1 \\
\pm 21 \mathrm{~mm} \mathrm{Hg} \\
\text { Diastolic BP } \\
\text { RD group }(n=52) \text { : } \\
\text { Baseline } 178 \pm 17 / 98 \pm 16 \mathrm{~mm} \mathrm{Hg} \text {. Change at } \\
1 \text { months }-7,3 \text { months }-8,6 \text { months }-12 \\
\pm 11 \text { mm Hg. } \\
\text { Control group }(n=54) \text { : Baseline mm Hg. Change } \\
\text { at } 1 \mathrm{months} 0,3 \text { months }-2,6 \text { months } 0 \\
\pm 10 \mathrm{~mm} \mathrm{Hg}\end{array}$ \\
\hline $\begin{array}{l}\text { SYMPLICITY } \\
\text { HTN-3 }{ }^{8} 9\end{array}$ & $\begin{array}{l}\text { Catheter-based RD with SYMPLICITY } \\
\text { Catheter System }\end{array}$ & Sham procedure & 1,6 months & $\begin{array}{l}\text { Systolic BP } \\
\text { RD group ( } n=364 \text { ): Baseline office } 179.7 \pm 16.1 \text {. } \\
\text { Value at } 6 \text { months: } 165.6 \pm 23.7 \text {. } \\
\text { Change from baseline: }-14.13 \pm 23.93 \mathrm{~mm} \mathrm{Hg} \\
\text { Baseline ambulatory: } 159.1 \pm 13.2 \text {. Value at } \\
6 \text { months: } 151.8 \pm 16.0 \text {. } \\
\text { Baseline home: } 169.0 \pm 15.9 \text {. Value at } 6 \text { months: } \\
161.1 \pm 19.2 \text {. } \\
\text { Control group ( } n=171 \text { ): Baseline office } 180.2 \\
\pm 16.8 \text {. Value at } 6 \text { months: } 168.4 \pm 28.6 \text {. } \\
\text { Change from baseline }-11.74 \pm 25.94 \mathrm{~mm} \mathrm{Hg} \\
\text { Baseline ambulatory: } 159.5 \pm 13.5 \text {. Value at } \\
6 \text { months: } 153.9 \pm 19.1 . \\
\text { Baseline home: } 169.1 \pm 16.3 \text {. Value at } 6 \text { months: } \\
162.8 \pm 21.1 \\
\text { Diastolic BP } \\
\text { RD group ( } n=364) \text { : Baseline office } 96.5 \pm 16.6 \text {. } \\
\text { Value at } 6 \text { months: } 89.5 \pm 16.9\end{array}$ \\
\hline
\end{tabular}


Observational studies

Brandt et al ${ }^{14} \quad$ RD with SYMPLICITY or Flex catheter (Ardian) with up to 6 ablations at $8 \mathrm{~W}$ for 2 min each were performed for both renal arteries

Brandt et $a l^{15} \quad$ RD with catheter (SYMPLICITY and Flex by Ardian) with up to 6 ablations at $8 \mathrm{~W}$ for 2 min each were performed for both renal arteries
Details of control group not specified

1 and 6 months

Details of control group not specified

1,3 and 6 months

\section{Efficacy results}

Change from baseline $-6.75 \pm 15.11 \mathrm{~mm} \mathrm{Hg}$

Baseline ambulatory:88.0 \pm 14.0 . Value at 6 months: $83.1 \pm 13.7$.

Baseline home: $89.6 \pm 15.9$. Value at 6 months: 86.0 \pm 16.6 .

Control group $(n=171)$ : Baseline office 98.9

\pm 15.8 . Value at 6 months: $94.1 \pm 17.7$

Change from baseline $-4.79 \pm 17.25 \mathrm{~mm} \mathrm{Hg}$.

Baseline ambulatory: $90.9 \pm 14.4$. Value at

6 months: $87.4 \pm 14.6$

Baseline home: $92.9 \pm 16.4$. Value at 6 months: $90.0 \pm 16.4$

Resting systolic BP

RD group $(n=46)$ : Baseline 180.7 $\pm 18.3,1$ month

$158.2 \pm 17.6,6$ months $152.9 \pm 22.4 \mathrm{~mm} \mathrm{Hg}$

Control group $(n=18)$ : Baseline 184.5 \pm 22.1 ,

1 month $181.6 \pm 26.3,6$ months 182.8

$\pm 24.6 \mathrm{~mm} \mathrm{Hg}$.

Resting diastolic BP

RD group $(n=46)$ : Baseline 95.8 $\pm 10.1,1$ month

$88.6 \pm 10.9,6$ months $87 \pm 12.9 \mathrm{~mm} \mathrm{Hg}$.

Control group $(n=18)$ : Baseline 98.2 \pm 13.6 ,

1 month $98 \pm 12.7,6$ months $99.8 \pm 16.5 \mathrm{~mm} \mathrm{Hg}$.

The average number of antihypertensives was constant for control group $4.8 \pm 2.5$ while in $\mathrm{RD}$

group it deceased in seven patients (15\%) which led to a change from $4.7 \pm 0.5$ to $4.5 \pm 1.6$

antihypertensives

Resting systolic BP

RD group $(n=110)$ : Baseline 181 $\pm 24.7,1$ month

$161.1 \pm 22.8,3$ month $159.1 \pm 22.1,6$ months

$152.1 \pm 20 \mathrm{~mm} \mathrm{Hg}$. Control group $(n=10)$ :

Baseline 183.9 $\pm 21.6,1$ month $181.3 \pm 18.5$,

3 month $190.6 \pm 16.9,6$ months 193.9

$\pm 15.4 \mathrm{~mm} \mathrm{Hg}$

Resting diastolic BP

RD group $(n=110)$ : Baseline $91.4 \pm 12.8,1$ month

$87 \pm 14,3$ months $84 \pm 13.1,6$ months 83.7

$\pm 13.5 \mathrm{~mm} \mathrm{Hg}$. Control group $(n=10)$ : Baseline

Continued 


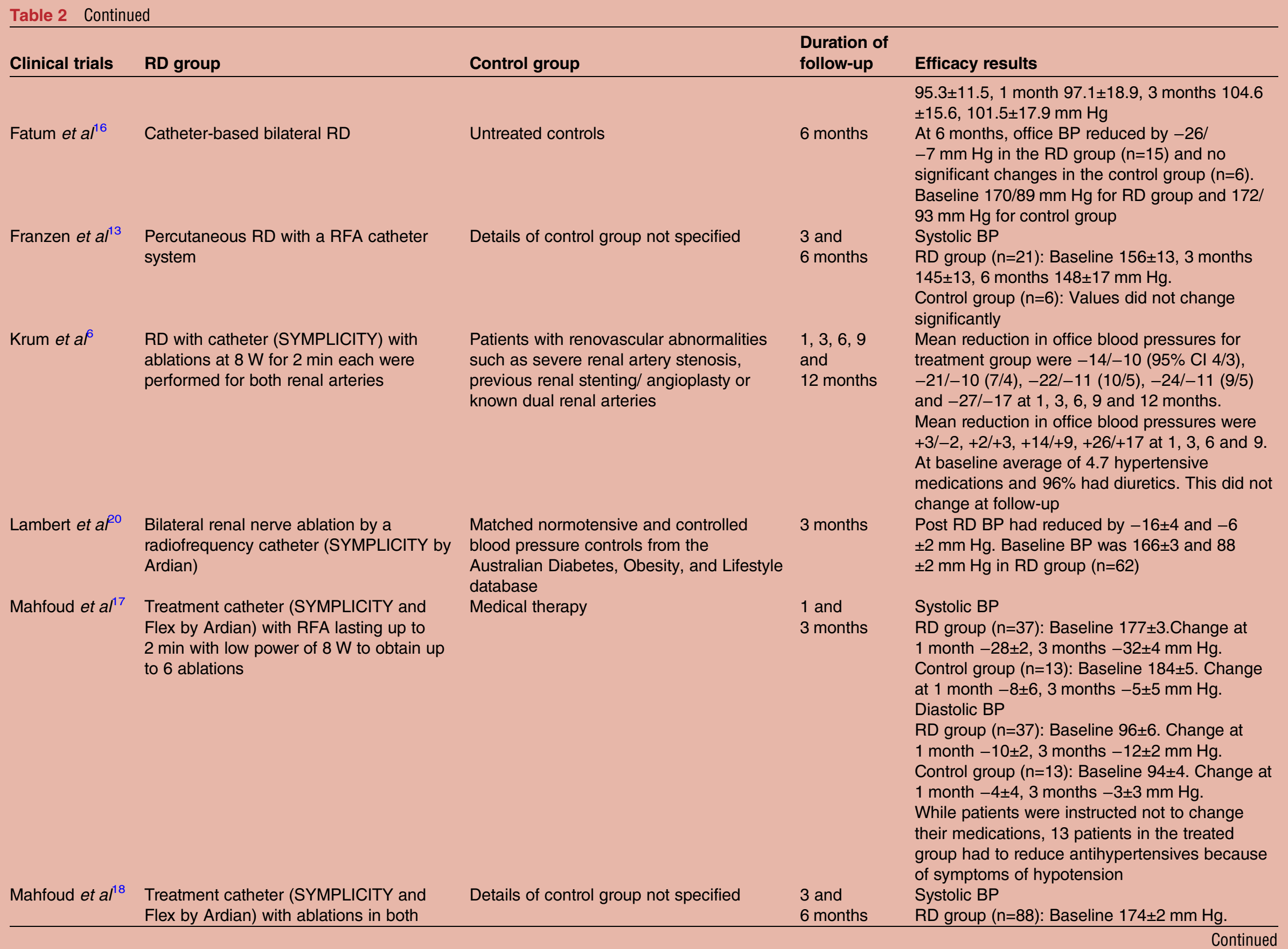


Table 2 Continued

\begin{tabular}{|c|c|c|c|c|}
\hline Clinical trials & RD group & Control group & $\begin{array}{l}\text { Duration of } \\
\text { follow-up }\end{array}$ & Efficacy results \\
\hline & $\begin{array}{l}\text { renal arteries with up to } 8 \text { ablations for } \\
2 \text { min with a maximum of } 8 \mathrm{~W}\end{array}$ & & & $\begin{array}{l}\text { Change at } 3 \text { month }-22.7 \pm 2.3,6 \text { months }-26.6 \\
\pm 2.5 \mathrm{~mm} \mathrm{Hg} \text {. Control group }(\mathrm{n}=12) \text { : Baseline } 184 \\
\pm 7 \mathrm{~mm} \mathrm{Hg} \text {. Change at } 3 \mathrm{month}-7.2 \pm 7.6 \text {, } \\
6 \text { months }-4.4 \pm 6.2 \mathrm{~mm} \mathrm{Hg} \text {. } \\
\text { Diastolic BP } \\
\text { RD group ( } \mathrm{n}=88 \text { ): Baseline } 95 \pm 2 \mathrm{~mm} \mathrm{Hg} \text {. } \\
\text { Change at } 3 \text { month }-7.7 \pm 1.3,6 \text { months }-9.7 \\
\pm 1.5 \mathrm{~mm} \mathrm{Hg} \text {. Control group }(\mathrm{n}=12) \text { : Baseline } 97 \\
\pm 5 \mathrm{~mm} \mathrm{Hg} \text {. Change at } 3 \mathrm{month}-4.1 \pm 4.7 \text {, } \\
6 \mathrm{months}-3.0 \pm 4.3 \mathrm{~mm} \mathrm{Hg} \text {. } \\
\text { Number of antihypertensives was } 5.7 \pm 0.2 \text {. At } \\
3 \text { months follow-up } 18 \text { patients }(18 \%) \text { had } \\
\text { reduced antihypertensives use and } 7(7 \%) \text { had } \\
\text { increased antihypertensive use }\end{array}$ \\
\hline Ukena et al ${ }^{19}$ & $\begin{array}{l}\text { Catheter-based RD with SYMPLICITY } \\
\text { Catheter System }\end{array}$ & Details of control group not specified & 3 months & $\begin{array}{l}\text { Systolic BP } \\
\text { RD group ( } n=37) \text { : Baseline } 172 \pm 24,3 \text { months } \\
141 \pm 21 \mathrm{~mm} \mathrm{Hg} \text {. Change at } 3 \text { months } 31 \\
\pm 13 \mathrm{~mm} \mathrm{Hg} \text {. Control group }(n=9) \text { : Baseline } 166 \\
\pm 23,3 \mathrm{months} 166 \pm 25 \mathrm{~mm} \mathrm{Hg} \text {. Change at } \\
3 \text { months } 0 \pm 17 \mathrm{~mm} \mathrm{Hg} \text {. } \\
\text { Diastolic BP } \\
\text { RD group ( } n=37) \text { : Baseline } 94 \pm 19,3 \text { months } 85 \\
\pm 16 \mathrm{~mm} \mathrm{Hg} \text {. Change at } 3 \text { months: }-9 \\
\pm 13 \mathrm{~mm} \mathrm{Hg} \text {. Control group ( } \mathrm{n}=9 \text { ): Baseline } 90 \pm 7 \text {, } \\
3 \mathrm{months} 89 \pm 9 \mathrm{~mm} \text { Hg. Change at } 3 \text { months }-1 \\
\pm 5 \mathrm{~mm} \mathrm{Hg}\end{array}$ \\
\hline
\end{tabular}




\section{A) Systolic blood pressure}

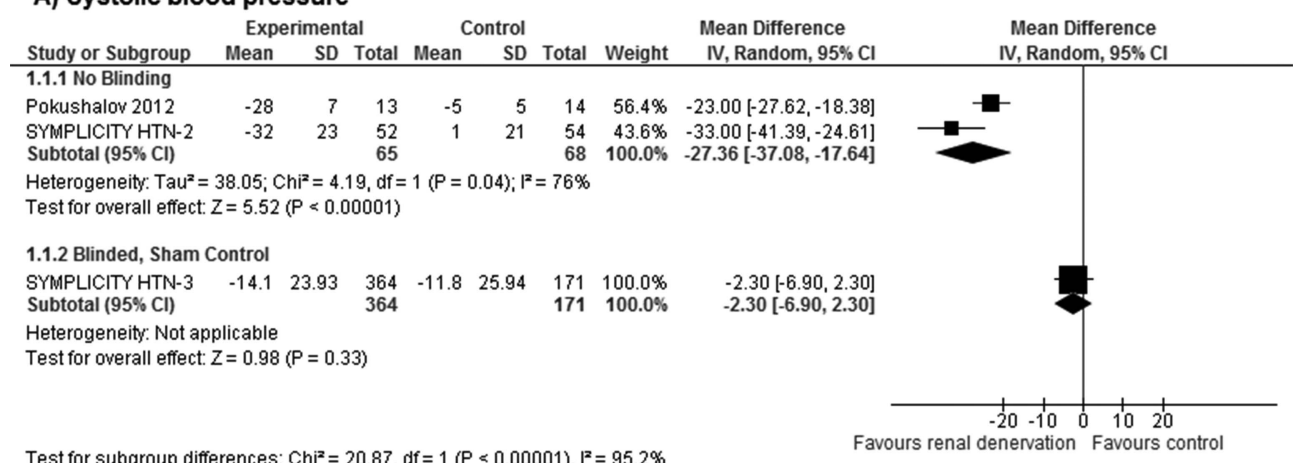

Test for subaroup differences: $\mathrm{Chi}^{2}=20.87, \mathrm{df}=1(\mathrm{P}<0.00001) . \mathrm{l}^{2}=95.2 \%$

\section{B) Diastolic blood pressure}

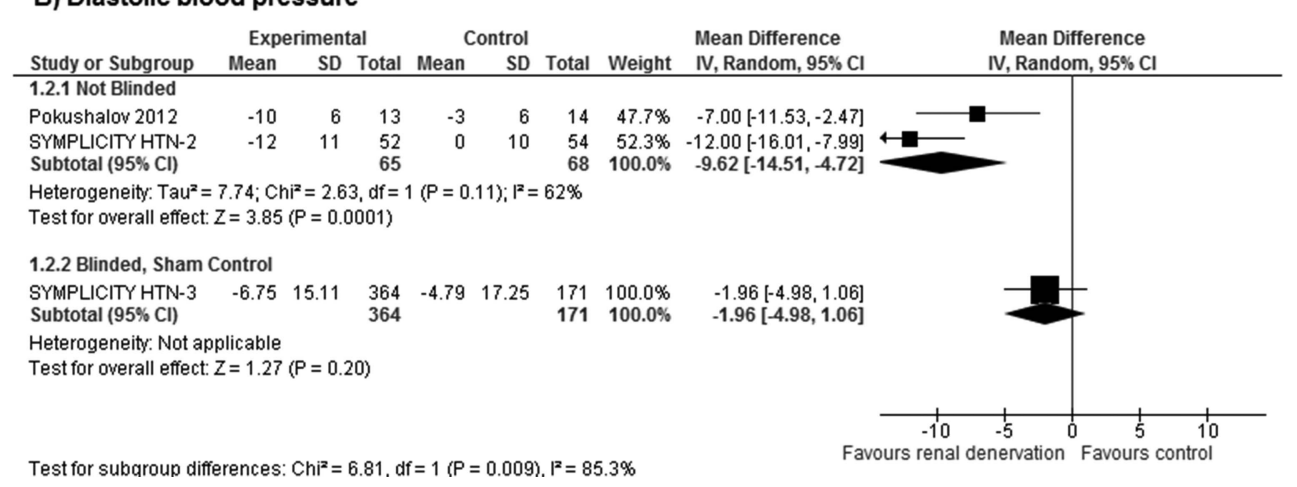

Figure 1 Mean difference in systolic and diastolic blood pressure for the three randomised controlled trials at follow-up postrenal denervation.

data from two unblinded trials of lower quality showed significant reduction in change in systolic $(-27.3695 \%$ CI -37.08 to $-24.61 \mathrm{~mm} \mathrm{Hg}$ ) and diastolic blood pressure $(-9.6295 \%$ CI -14.51 to $-4.72 \mathrm{~mm} \mathrm{Hg})$. The subgroup testing proves significant differences between the two data set and confirms that moderate-high risk of bias studies give significant effects while low risk of bias studies do not. The results are presented in figure 1.

Results from other observational studies are shown in table 2. Systolic and diastolic blood pressure reductions with renal denervation were reported by all of these studies. ${ }^{6}{ }^{13-20}$

Only a few studies reported adverse events associated with renal denervation (see online supplementary appendix 4). The SYMPLICITY HTN-3 trial was the only study to report cardiovascular events at 6 months follow-up. There was no significant difference between treatment and control group in terms of death, myocardial infarction, new onset renal disease, stroke and hypertensive crisis or emergency. The most commonly reported adverse event was a pseudoaneurysm at the femoral access site and this was reported in four studies. ${ }^{6} 71718$ Other adverse events reported included renal artery dissection, ${ }^{6}$ contrast medium allergic reaction, ${ }^{18}$ postprocedural hypotension, ${ }^{7}$ intraprocedural bradycardia ${ }^{7}$ and five cases of hypertensive emergencies requiring admission to hospitalisation (three in the renal denervation group and two in the control group) ${ }^{7}$

\section{DISCUSSION}

Our systematic review of three randomised controlled trials with 688 participants has suggested a wide range of reported outcomes associated with the treatment of drug-resistant hypertension by renal denervation. Data derived from the highest quality single-blind, randomised, sham-controlled trial (SYMPLICITY HTN-3) suggests that renal denervation does not produce significant reductions in systolic blood pressure and diastolic blood pressure while lower quality non-blinded randomised controlled trials and observational studies suggest that there are significant reductions in blood pressure with renal denervation. These latter studies are confounded by significant bias.

The choice of control arm is an important consideration when evaluating studies of renal denervation. The SYMPLICITY HTN-3 trial was the first randomised controlled trial to use both a sham-control group and blinding. This raises the issue of possible bias during outcome assessment in the other two randomised controlled trials and non-blinded observational studies where there may have been major differences in the subsequent monitoring and follow-up, or medication adherence and use of cointerventions among patients who had undergone renal denervation. Inadequate blinding may cause bias through differences in recording blood pressure. Interestingly, in the SYMPLICITY studies, the magnitude of reduction in blood pressure following renal 
denervation was also significantly greater if recorded through office measurements compared to ambulatory measurements. For example, the decrease in systolic blood pressure at 6 months following renal denervation was between 25 and $30 \mathrm{~mm} \mathrm{Hg}$ for office blood pressure measurements while on $24 \mathrm{~h}$ ambulatory monitoring, it was approximately $10 \mathrm{~mm} \mathrm{Hg} .{ }^{6} 721$ The reasons for these differences in magnitude of benefit depending on the modality by which blood pressure is measured remain unclear although ambulatory blood pressure monitors operate in a blinded fashion whereas office blood pressure measurements as in the SYMPLICITY studies are recorded in an unblinded fashion and were therefore subject to bias. The failure of the SYMPLICITY HTN-3 trial to meet the primary blood pressure end point is of interest. The blinded, sham nature of the study would remove many of the biases included in unblinded denervation studies, such as the possibility of decreased medication adherence in patients randomised to the medical treatment arm with improved adherence in the treatment arm. Office-based blood pressure measurements are a significant source of major bias, ${ }^{22}$ with significant potential for regression towards the mean. Patients enrolled in hypertension studies have a better chance of meeting the inclusion criteria of the study on a day when their blood pressure is above their own long-term mean. If the patient's blood pressure is then followed up, its average will tend to return to that individual's true mean pressure, even if there was no intervention introducing bias. ${ }^{22}$ One aspect that has drawn comment regarding the failure of the SYMPLICITY HTN-3 trial to show efficacy in the treatment of drug-resistant hypertension relates to whether or not participants achieved optimal renal denervation and the potential for different subgroups of patients to respond differently to the denervation procedure. ${ }^{23-25}$ Reductions in blood pressure were observed in specific subgroups of patients particularly in non-black patient cohorts and in younger patients, although the absolute reduction in BP in these subgroups was small with an order of magnitude around $5-6 \mathrm{~mm} \mathrm{Hg}$. Whether this represents differences in the importance of the sympathetic nerve system in the pathophysiology of drug-resistant hypertension in these cohorts or the efficacy of the procedure in disrupting neural pathways in these patients remains unclear.

While angiographic markers of successful delivery of energy causing vascular disruption (notching) can be seen during the denervation procedure, no reliable markers of renal denervation are available, hence the completeness and extent of renal nerve disruption is uncertain. The unipolar nature of the SYMPLICITY system used in this trial makes it more technically challenging to ensure true circumferential ablation, compared to more contemporary multipolar systems, hence the efficacy of the treatment will be highly operator dependent. Interestingly, the SYMPLICITY HTN-3 trial reported that outcomes between operators performing five or more procedures and those performing fewer than five procedures were similar, with no evidence of a learning curve for high-volume operators when earlier procedures were compared with later ones. It remains unclear whether similar results will be seen with other multipolar systems currently on the market, which have been designed to enable the delivery of a circumferential ablation to the renal artery, or whether the lack of efficacy reported with the SYMPLICITY system represents a class effect. An interesting finding of the SYMPLICITY HTN-3 was that major reductions in blood pressure, which were not present in the previous SYMPLICITY trials, were observed in the control group. One explanation for this was that there was greater exposure to spironolactone in SYMPLICITY HTN $-3{ }^{25}$ The other explanations may relate to the difference in control arm and the use of sham operation, which may lead to improved adherence to medication and the placebo effect.

Despite renal denervation being undertaken predominantly in patients with drug-resistant hypertension who are at significant risk from future cardiovascular events, the very nature of an invasive procedure itself may predispose to potential cardiovascular complications. SYMPLICITY HTN-3 is the first high-quality study to demonstrate the safety of the procedure in terms of cardiovascular disease and mortality end points, with no significant increases in major adverse events, defined as death from any cause, end-stage renal disease, embolic events resulting in end-organ damage, renal-artery or other vascular complications, hypertensive crisis within 30 days or new renal-artery stenosis of more than $70 \%$ within 6 months following the denervation procedure. A few other studies have described procedure-related complications, the most common of which being access site related complications at the femoral access such as haematomas, pseudoaneurysms, etc (approximately $2 \%$ ), with two reported cases of renal artery dissection $(<1 \%)$ that needed renal artery stenting. ${ }^{6} 2126$ Furthermore, concerns have been raised regarding the impact of renal denervation on renal function. ${ }^{27}$ There is limited long-term evidence from the SYMPLICITY HTN-1, that there was a decrease in estimatedglomerular filtration rate after renal denervation but it is unclear if this is related to changes in medication after the procedure. ${ }^{28}$

Our systematic review has a few strengths. We were able to identify studies that reported mean differences and SDs that enable statistical pooling of results. We have considered studies of different design that varied in their risk of bias. Our systematic review relies on the most up-to-date evidence, including data derived from the largest, highest quality randomised controlled trial.

\section{Study limitations}

The major limitations of our review stem from only one high-quality study; the majority were underpowered and longer follow-up is needed. Furthermore, there may have been overlap of some of the studies, for example, 
both the Ukena ${ }^{19}$ and Mahfoud ${ }^{17} 18$ analyses contained a small number of patients that were enrolled as part of the SYMPLICITY HTN-1 and/or SYMPLICITY HTN-2 studies. The sample sizes of studies included were as small as 20 patients, which are insufficient to capture safety events. Non-blinded studies have a risk of bias in outcome assessment and the heterogeneity and lack of management description of the control group is another limitation. These studies lack a standardised diagnostic work-up to exclude secondary hypertension and do not include ambulatory out-of-the-office blood pressure measurement to exclude white coat hypertension or a formal assessment of adherence. Finally, there is the potential for publication bias and selective outcome reporting, particularly with a new technology such as renal denervation where investigators who found no benefit from renal denervation may have decided either to not publish the data or to only report selected significant findings.

In summary, evidence for the efficacy of renal denervation using catheter-based systems in reducing blood pressure in resistant hypertension is derived from unblinded studies that are at risk of bias. The highest quality single blinded randomised controlled trial did not show efficacy in office blood pressure reduction, although it did meet its safety end point. Future studies investigating the efficacy of renal denervation in the treatment of drug-resistant hypertension should be undertaken in a blinded manner, with sham procedures in the control group and ambulatory monitoring to reduce the potential for bias.

Contributors CSK and MAM conceptualised the review and developed the protocol. CSK and YKL analysed the data. CSK, YKL and MAM wrote the manuscript. BK and ME- 0 contributed to the writing of the manuscript. CSK and SP abstracted the data, which was checked by YKL and MAM. CSK and MAM will act as guarantors for the paper.

Funding CSK is an Academic Clinical Fellow in Cardiology and is funded by the National Institute for Health Research.

Competing interests None.

Provenance and peer review Not commissioned; internally peer reviewed.

Data sharing statement No additional data are available.

Open Access This is an Open Access article distributed in accordance with the Creative Commons Attribution Non Commercial (CC BY-NC 3.0) license, which permits others to distribute, remix, adapt, build upon this work noncommercially, and license their derivative works on different terms, provided the original work is properly cited and the use is non-commercial. See: http:// creativecommons.org/licenses/by-nc/3.0/

\section{REFERENCES}

1. World Health Organization. Global health risks: mortality and burden of disease attributable to selected major risks. 2009.

2. Hajjar I, Kotchen TA. Trends in prevalence, awareness, treatment, and control of hypertension in the United States, 1988-2000. JAMA 2003;290:199-206.

3. Calhoun DA, Jones D, Textor S, et al. American Heart Association Professional Education Committee. Resistant hypertension: diagnosis, evaluation, and treatment. A scientific statement from the American Heart Association Professional Education Committee of the Council for High Blood Pressure Research. Hypertension 2008;51:1403-19.

4. Schlaich M, Sobotka P, Krum H, et al. Renal denervation as a therapeutic approach for hypertension: novel implications for an old concept. Hypertension 2009;54:1195-201.

5. Doumas M, Douma S. Renal sympathetic denervation: the jury is still out. Lancet 2010;376:1878-80.

6. Krum H, Schlaich M, Whitbourn R, et al. Catheter-based renal sympathetic denervation for resistant hypertension: a multicentre safety and proof-of-principle cohort study. Lancet 2009;373:1275-81

7. Symplicity HTN-2 Investigators. Renal sympathetic denervation in patients with treatment-resistant hypertension (The Symplicity HTN-2 Trial): a randomised controlled trial. Lancet 2010;376:1903-9.

8. Kandzari DE, Bhatt DL, Sobotka PA, et al. Catheter-based renal denervation for resistant hypertension: rational and design of the SYMPLICITY HTN-3 trial. Clin Cardiol 2012;35:528-35.

9. Bhatt DL, Kandzari DE, O'Neill WW, et al. SYMPLICITY HTN-3 Investigators. A controlled trial of renal denervation for resistant hypertension. N Engl J Med 2014;370:1393-401.

10. Higgins JPT, Deeks JJ, Altman DG. Chapter 9: analysing data and undertaking meta-analyses. In: Higgins JPT, Green S, eds. Cochrane handbook for systematic reviews of interventions. Chichester, UK: John Wiley \& Sons, 2008.

11. Ioannidis JP, Trikalinos TA. The appropriateness of asymmetry tests for publication bias in meta-analyses: a large survey. CMAJ 2007;176:1091-6.

12. Pokushalov E, Romanov A, Corbucci G, et al. A randomized comparison of pulmonary vein isolation with versus without concomitant renal artery denervation in patients with refractory symptomatic atrial fibrillation and resistant hypertension. J Am Coll Cardiol 2012;60:1163-70.

13. Franzen KF, Mortensen K, Himmel F, et al. Percutaneous renal denervation improves central hemodynamics and arterial stiffnessa pilot study. ESC Congress; Vol 33, Munchen, Germany 2012:771.

14. Brandt MC, Mahfoud F, Reda S, et al. Renal sympathetic denervation reduces left ventricular hypertrophy and improves cardiac function in patients with resistant hypertension. $\mathrm{J} \mathrm{Am} \mathrm{Coll}$ Cardiol 2012;59:901-9.

15. Brandt MC, Reda S, Mahfoud F, et al. Effects of renal sympathetic denervation on arterial stiffness and central hemodynamics in patients with resistant hypertension. J Am Coll Cardiol 2012;60:1956-65.

16. Fatum C, Gisbertz C, Himmel F, et al. Effect of renal denervation therapy on sodium excretion in patients with resistant hypertension. ESC Congress; Vol 33, Munchen, Germany, 2012:182.

17. Mahfoud F, Schlaich M, Kindermann I, et al. Effect of renal sympathetic denervation on glucose metabolism in patients with resistant hypertension. Circulation 2011;123:1940-6.

18. Mahfoud F, Cremers B, Janker J, et al. Renal hemodynamics and renal function after catheter-based renal sympathetic denervation in patients with resistant hypertension. Hypertension 2012;60:419-24.

19. Ukena C, Mahfoud F, Kindermann I, et al. Cardiorespiratory response to exercise after renal sympathetic denervation in patients with resistant hypertension. J Am Coll Cardiol 2011;58:1176-82.

20. Lambert GW, Hering D, Esler MD, et al. Health-related quality of life after renal denervation in patients with treatment-resistant hypertension. Hypertension 2012;60:1479-84.

21. Symplicity HTN-1 Investigators. Catheter-based renal sympathetic denervation for resistant hypertension: durability of blood pressure reduction out to 24 months. Hypertension 2011:57:911-17.

22. Howard JP, Nowbar AN, Francis DP. Size of blood pressure reduction from renal denervation: insights from meta-analysis of antihypertensive drug trials of 4121 patients with focus on trial design: the CONVERGE report. Heart 2013;99:1579-87.

23. Bilazarian S. Confession of a SYMPLICITY HTN-3 Trialist. Medscape, 2014

24. Bakris G. The SYMPLICITY HTN-3 Co-PI gives his 2 cents Medscape, 2014.

25. Messerli FH, Bangalore S. Renal denervation for resistant hypertension? N Engl J Med 2014;370:1454-7.

26. Symplicity HTN-2 Investigators. Renal sympathetic denervation in patients with treatment-resistant hypertension (The Symplicity HTN-2 Trial): a randomised controlled trial. Lancet 2010;376:1903-9.

27. Uder A, Schmid A, Titze S, et al. Renal artery denervation for the treatment of hypertension: opening up new horizons. Cardiovasc Intervent Radiol 2011;34:442-4.

28. Mafeld S, Vasdev N, Haslam P. Renal denervation for treatment-resistant hypertension. Ther Adv Cardiovasc Dis 2012;6:245-58. 